\title{
Far-Field Noise Minimization Using an Adjoint Approach
}

\author{
Markus P. Rumpfkeil and David W. Zingg \\ University of Toronto Institute for Aerospace Studies \\ 4925 Dufferin Street, Toronto, Ontario, M3H 5T6, Canada \\ markus@oddjob.utias.utoronto.ca
}

\section{Introduction}

In this paper, shape optimization is applied to a blunt trailing edge airfoil in an unsteady turbulent flow environment to minimize the radiated far-field noise using a novel hybrid Unsteady Reynolds-Averaged Navier-Stokes/Ffowcs Williams and Hawkings (URANS/FW-H) optimization algorithm. Airframegenerated noise is an important component of the total noise radiated from commercial aircraft, especially during aircraft approach and landing, when engines operate at reduced thrust, and airframe components (such as high-lift devices) are in the deployed state [SLL03]. Future Federal Aviation Administration noise regulations, the projected growth in air travel, and the increase in population density near airports will require future civil aircraft to be substantially quieter than the current ones. Consequently, the attempt to understand and reduce airframe noise has become an important research topic [SG04].

A typical approach to tackle airframe-generated noise computations is to represent the CFD solution on a reasonable computational mesh that does not extend too far from the aircraft. A near-field plane or surface within the computational mesh can then serve as an interface between the CFD solution and a wave propagation program based on principles of geometrical acoustics and nonlinear wave propagation [SLL03]. The two-dimensional Ffowcs Williams and Hawkings (FW-H) equation in the frequency-domain [Loc00] is the wave propagation formulation of choice in this work.

This paper employs a general framework to derive a discrete adjoint method for the optimal control of unsteady flows [RZ07, RZ08] together with a Newton-Krylov approach to optimization [NZ02, NZ04]. We present several design problems using the novel hybrid URANS/FW-H optimization algorithm after validating the $\mathrm{FW}-\mathrm{H}$ wave propagation code in the next section.

\section{Noise Prediction Validation}

A direct comparison between the pressure fluctuations calculated via the FW-H approach and those obtained from a CFD simulation is performed at three distinct locations with increasing distance from an airfoil. The laminar 
flow over the single-element NACA 0012 airfoil with a Reynolds number of 800, a free stream Mach number of 0.2 and an angle of attack of $20^{\circ}$ is considered. At these conditions the airfoil experiences vortex shedding. A C-mesh with $848 \times 395$ nodes and a non-dimensional time step $\Delta t=0.03$ is used. After the flow solver has reached a periodic steady state, 1800 time steps are taken, which cover about five vortex shedding cycles, and the solution is recorded.

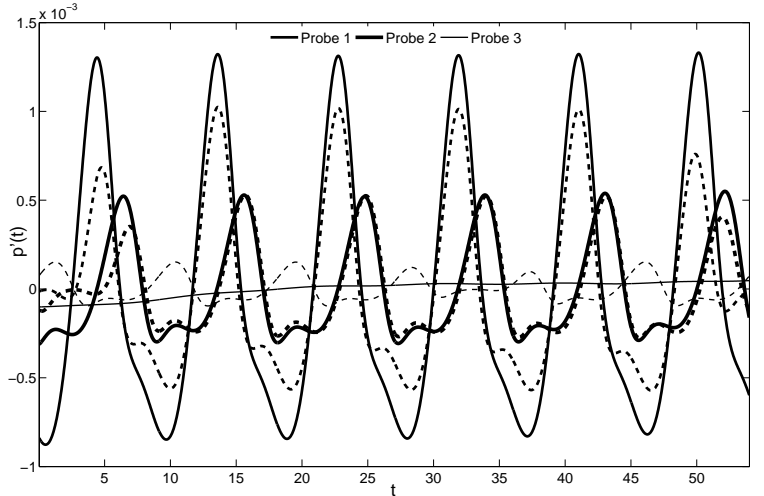

Fig. 1: Comparison of pressure fluctuations calculated by CFD (solid) and FW-H (dashed).

The extracted CFD pressure fluctuations and those computed using the FW-H solver are plotted in Figure 1 for the three probe locations. In two dimensions one expects that the sound intensity, which itself is proportional to the square of the sound pressure, is inversely proportional to the distance of an acoustic point source. This distance law is almost perfectly fulfilled by the pressure fluctuations which are calculated with the FW-H approach.

Comparing the pressure fluctuations probe location by probe location one can make the following observations: At probe location 2, which is about $2 c$ below the airfoil, the two pressure records are almost identical, except for the beginning and end of the data, where the necessary window function [Loc00] tarnishes the result from the FW-H approach. The agreement in the first probe location (less than $1 c$ below) is also fairly good, except for the overprediction of the amplitude by the CFD calculation. The CFD results at probe location 3 (30c below) are basically useless due to the coarser grid this far away.

\section{Results}

A NACA 0012 airfoil with a $0.03 c$ thick blunt trailing edge in a turbulent flow is investigated. The free-stream flow conditions are given by $M_{\infty}=0.2$, $R e=2 \times 10^{6}, \alpha=0^{\circ}$, and the mesh consists of about 36,000 nodes. As displayed in Figure 2, the comparison of pressure fluctuations calculated by CFD and FW-H for the initial airfoil show good agreement at a location about $\frac{1}{3} c$ below the trailing edge of the initial airfoil.

First, a remote inverse shape design problem is studied with a discrete cost function $J$ given by

$$
J=\frac{1}{2} \Delta t \sum_{\text {timesteps }}\left(p_{o b s}^{n}-p_{o b s}^{* n}\right)^{2} .
$$




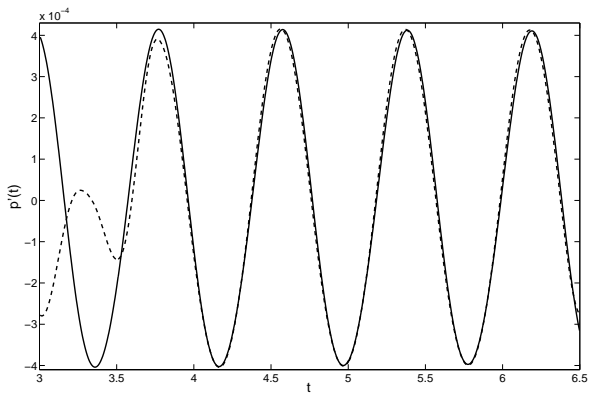

Fig. 2: Comparison of pressure fluctuations calculated by CFD (solid) and FW-H (dashed) about $\frac{1}{3} c$ below the trailing edge of initial airfoil.

Here, $p_{o b s}^{n}$ is the pressure at some far-field observer location at time step $n$ obtained from a current airfoil shape, and $p_{o b s}^{* n}$ is the target pressure at the same location and time step obtained from the target airfoil shape, which is given through a perturbation in two shape design variables of the initial blunt NACA 0012 airfoil. Both airfoils are shown in Figure 3 and the far-field observer location is $40 \mathrm{c}$ below the trailing edge.

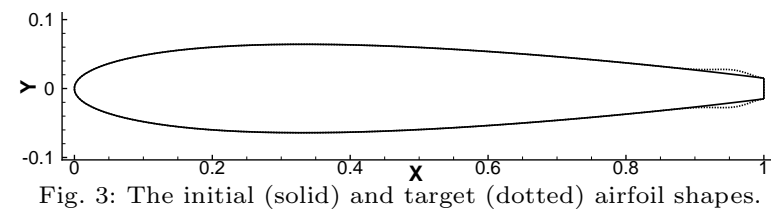

In order to reduce the computational costs in the actual optimization runs a bigger non-dimensionalized time step of $\Delta t_{c}=0.01$ is utilized for the first $N_{c}=300$ steps for the adjustment period after a shape modification has taken place, which can be seen in Figure 8. Once the interval where the pressures are compared is reached, a smaller time step $\Delta t=0.005$ is used for another 700 steps, leading to $N=1000$ time steps in total for each flow solve covering a time interval of $[0,6.5]$. The corresponding adjoint equations for this situation are given in Rumpfkeil and Zingg [RZ07, RZ08].

The convergence history of this remote inverse shape design problem with the adjoint approach in comparison to a second-order central finite-difference approach with a step size of $h=10^{-5}$ is shown in Figure 4. The objective function $J$ is always scaled such that its initial value is unity. One can see that the objective functions are driven to small values in about ten design iterations and that the two approaches show a reasonable agreement, which means that the adjoint approach for the gradient calculation is accurate. For example, at the first design iteration the finite difference method $(f d)$ and adjoint method $(a d)$ yield

$$
\left(\frac{\partial J}{\partial Y}\right)_{f d}=(-33.53,34.18), \quad\left(\frac{\partial J}{\partial Y}\right)_{a d}=(-34.36,35.11) .
$$

The computational time of a gradient computation is about two to three times the time of a flow solve, since the non-linear flow solve problem has a much 
better initial guess leading to less linear iterations per time step than the linear adjoint problem.

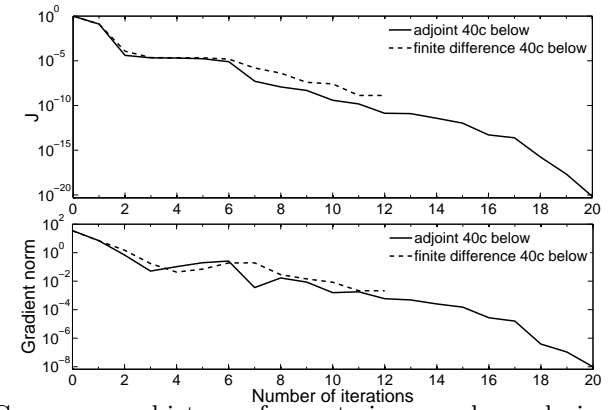

Fig. 4: Convergence history of remote inverse shape design problem.

Next, two more practically relevant optimizations are considered with the same flow conditions but two different objective functions:

$$
\begin{array}{ll}
\text { 1. Mean drag } & J_{D}=\bar{C}_{D}=\frac{1}{\# \text { time steps }} \sum_{\text {timesteps }} C_{D}^{n} \\
\text { 2. Pressure fluctuations } & J_{N}=\sum_{\text {timesteps }}\left(p_{\text {obs }}^{n}-\bar{p}_{\text {obs }}\right)^{2}=\sum_{\text {timesteps }}\left(p_{\text {obs }}^{n}\right)^{2}
\end{array}
$$

where $\bar{p}_{\text {obs }}$ is the mean pressure in the observer location, which is located $40 c$ below the trailing edge, $p_{o b s}^{\prime n}=p_{o b s}^{n}-\bar{p}_{o b s}$ is the pressure fluctuation in the observer location, and $C_{D}^{n}$ is the drag coefficient both at time step $n$.

Eight B-spline control points are used as shape design variables which are all located in the aft 15 percent of the chord length (four on the upper and four on the lower surface). The unsteady shape optimizations are started from three different initial shapes, which are shown in Figure 5 together with their initial objective function values:

1. The initial airfoil (solid)

2. The airfoil that results from setting all eight design variables to their specified upper bound (dashed)

3. The airfoil that results from setting all eight design variables to their specified lower bound (dotted)

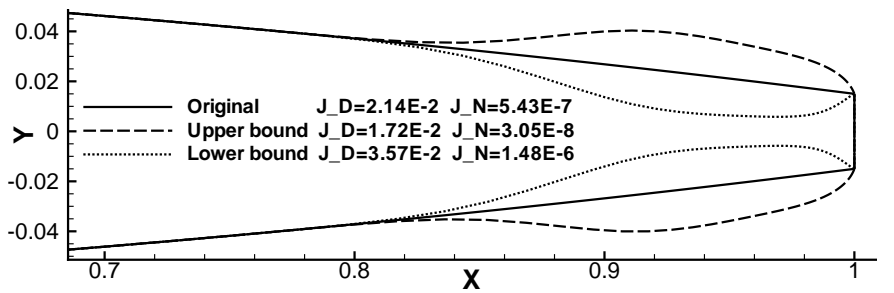

Fig. 5: Initial shapes for the turbulent blunt trailing edge flow optimizations.

The time horizon and time step sizes are the same as used in the remote inverse shape design presented earlier in this section. Figure 6 presents the final optimized airfoil shapes together with their objective function values. All three initial shapes converge for each objective function to the same respective 
final shapes shown in dotted and dashed lines for the mean drag and noise minimizations, respectively. As indicated in the figure, the mean drag value of the noise minimized airfoil is slightly higher than the mean drag value of the mean drag minimized airfoil. Conversely, the pressure fluctuations of the mean drag minimized airfoil are a factor of two higher than the ones from the noise minimized airfoil. This shows that noise and drag improvements lead to qualitatively similar results to a first approximation, but they definitely do not yield the same optimized shapes.

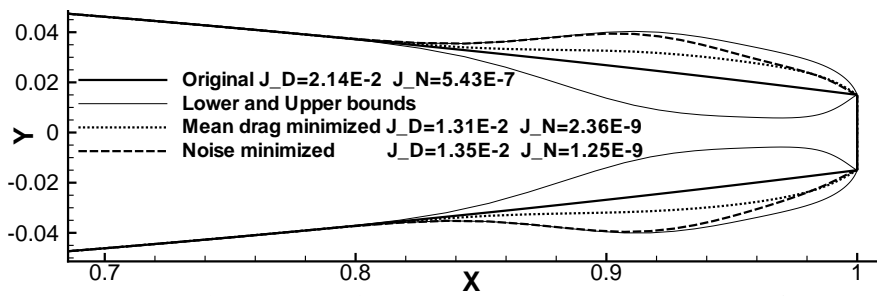

Fig. 6: Final improved airfoil shapes of the turbulent blunt trailing edge flow optimizations.

The convergence histories of the mean drag minimizations are displayed in Figure 7 (left). The objective function values are always scaled with the mean drag value of the original airfoil $J_{D}=2.14 \cdot 10^{-2}$ to make comparisons easier. Since all three initial shapes converge to the same final shape they have the same minimized objective function value which translates into a reduction in mean drag of about 39 percent from the original airfoil. The gradient norms are reduced by three to four orders of magnitude indicating that the optimizer has converged to a minimum in each case.
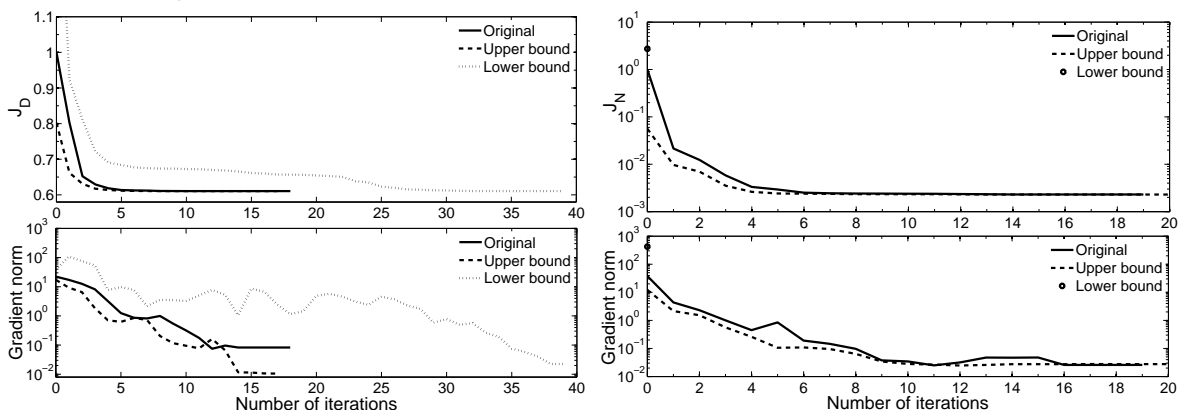

Fig. 7: Convergence histories of the mean drag (left) and noise minimizations (right).

The convergence histories of the noise minimizations (Figure 7 right) show that the sum of the squared pressure fluctuations for the optimized shape is reduced to 0.23 percent of the original airfoil's value $J_{N}=5.43 \cdot 10^{-7}$, which is again used to scale all the objective function values to ease comparisons. Starting from the lower bound leads to a failed line search in the first iteration because all gradients indicate that it would be beneficial to "slim" the airfoil even more which is not allowed by the box constraints imposed on the design variables to avoid grid movement and flow convergence problems. The gradient norms are reduced by two to three orders of magnitude. 

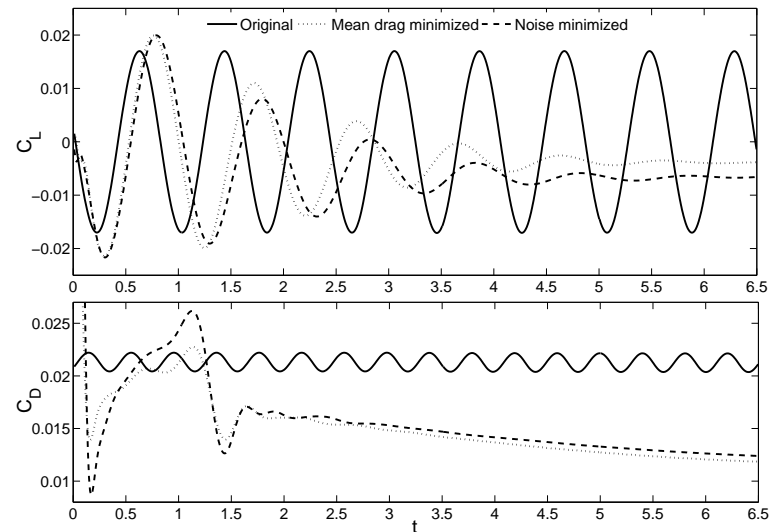

Fig. 8: Time histories of $C_{L}$ and $C_{D}$ before and after the optimizations vs. time ( $\left.\Delta t=0.005\right)$.

The time histories of $C_{L}$ and $C_{D}$ for the original blunt trailing edge airfoil before and after the optimizations are shown in Figure 8. One can clearly see the adjustment period for the improved airfoils in the time interval $[0,3]$ before they reach their new somewhat periodic steady state. A reduced mean drag for both optimized airfoils is also visible, and both objective functions lead to reduced oscillation amplitudes in both lift and drag.

\section{Conclusions}

The results presented in this paper show that the novel hybrid URANS/FW-H optimization algorithm, which uses a Newton-Krylov approach in combination with a discrete adjoint method, is effective and efficient for practical applications. We proved that it is possible to recover far-field pressure fluctuations via remote inverse shape designs in unsteady turbulent flows, and we were able to minimize the pressure fluctuations at a given far-field observer position in an efficient manner. Our future work will focus on the ability to modify a high-lift airfoil configuration to reduce the radiated noise while maintaining good aerodynamic performance.

\section{References}

[Loc00] Lockard, D.P.: An Efficient, Two-Dimensional Implementation of the FW-H Equation. J. of Sound and Vibration, 229(4), 897-911 (2000)

[NZ02] Nemec, M., Zingg, D.W.: Newton-Krylov Algorithm for Aerodynamic Design Using the Navier-Stokes Equations. AIAA J., 40(6), 1146-1154 (2002)

[NZ04] Nemec, M., Zingg, D.W.: Multipoint and Multi-Objective Aerodynamic Shape Optimization. AIAA J., 42(6), 1057-1065 (2004)

[RZ07] Rumpfkeil, M.P., Zingg, D.W.: A General Framework for the Optimal Control of Unsteady Flows with Applications. AIAA paper, 2007-1128

[RZ08] Rumpfkeil, M.P., Zingg, D.W.: The Optimal Control of Unsteady Flows with a Discrete Adjoint Method. Optimization and Engineering, doi:10.1007/s11081-008-9035-5 (2008)

[SG04] Singer, B.A., Guo, Y.: Development of Comp. Aeroacoustics Tools for Airframe Noise Calculations. Int. J. of CFD, 18(6), 455-469 (2004)

[SLL03] Singer, B.A, Lockard, D.P., Lilley G.M.: Hybrid Acoustic Predictions. Comp. and Math. with Applications, 46, 647-669 (2003) 